\title{
Exact solutions for steady-state, planar, magnetic reconnection in an incompressible viscous plasma
}

\author{
R. B. Fabling and I. J. D. Craig \\ University of Waikato, Hamilton, New Zealand
}

(Received 2 January 1996; accepted 26 January 1996)

\begin{abstract}
The exact planar reconnection analysis of Craig and Henton [Astrophys. J. 450, 280 (1995)] is extended to include the finite viscosity of the fluid and the presence of nonplanar components in the magnetic and velocity fields. It is shown that fast reconnection can be achieved for sufficiently small values of the kinematic viscosity. In particular, the dissipation rate is sustained by the strong amplification of planar magnetic field components advected toward the neutral point. By contrast, nonplanar field components are advected without amplification and so dissipate energy at the slow Sweet-Parker rate. () 1996 American Institute of Physics. [S1070-664X(96)02806-6]
\end{abstract}

\section{INTRODUCTION}

Much effort has gone into finding steady-state, incompressible magnetic merging solutions. ${ }^{1,2}$ A prime motivation is understanding magnetic reconnection-a key process that allows topological change by cutting and rejoining field lines at null points in the field. It is believed, for example, that reconnection is an essential ingredient in the global magnetic collapse of the solar flare. ${ }^{3}$

Despite intensive theoretical investigation a convincing analytic model of reconnection has proved elusive. Analytic approaches fall into two broad categories depending on whether "open" or "closed" magnetic geometries are modeled. The recent generation of closed X-point solutions describe two-dimensional (2-D) planar reconnection in an arbitrarily compressible magnetic fluid. ${ }^{4}$ The reconnection region is bounded by a rigid, superconducting boundary that anchors the field. Small-amplitude disturbances dissipate remarkably quickly, confirming the possibility of fast reconnection-that is, reconnection at a rate that is practically independent of the plasma resistivity. ${ }^{5-7}$ Although the reconnection rate slows when finite compressibility and nonplanar field components are included in the model, this could simply be an artifact of the rigid boundary constraining the flow.

A complimentary approach is provided by studies of steady-state, incompressible reconnection in open geometries. Until recently, exact solutions were available only for antiparallel merging under the ansatz of 2-D "stagnation point flow." ${ }^{8-11}$ The absence of field line curvature implies that magnetic annihilation, as opposed to reconnection, is the mechanism of magnetic energy release.

In a recent analysis, Craig and Henton ${ }^{12}$-hereafter $\mathrm{CH}$-showed that exact reconnection solutions can be derived by considering sheared stagnation-point flows in open geometries. The shearing takes place across a narrow current layer that separates oppositely directed, curved, magnetic field lines. The analysis extends naturally into threedimensions (3-D), where solutions involve either current sheets ${ }^{13}$ or localized quasicylindrical current tubes aligned to the exhaust axis of the fluid. ${ }^{14}$ By contrast, the 2-D current structure always involves a single sheet aligned to one separatrix plane of the $\mathrm{X}$ point.
The main purpose of this paper is to expand the planar analysis of $\mathrm{CH}$ by incorporating the finite viscosity of the plasma. First, however, in Sec. II, we derive the equations of $2 \frac{1}{2}$-dimensional viscous reconnection. The exact inviscid solution is described in Sec. III, where it is noted that viscous effects are likely to be important in the region of high shear surrounding the neutral point. A detailed analysis of the viscous solution is described in Sec. IV. Our conclusions are summarized in Sec. V.

\section{THE PLANAR RECONNECTION SYSTEM}

\section{A. Steady-state equations}

We assume that the plasma is governed by the steadystate, incompressible resistive magnetohydrodynamic (MHD) equations. Adopting nondimensional variables, in which fluid velocities are expressed in units of the Alfvén speed at the boundary of the reconnection region, the momentum and induction equations may be written as

$$
\begin{aligned}
& (\mathbf{v} \cdot \boldsymbol{\nabla}) \mathbf{v}=\mathbf{J} \times \mathbf{B}-\boldsymbol{\nabla} P-\nu \boldsymbol{\nabla} \times \boldsymbol{\omega}, \\
& \mathbf{E}+\mathbf{v} \times \mathbf{B}=\eta \mathbf{J},
\end{aligned}
$$

where $\nu$ and $\eta$ are the dimensionless plasma viscosity and resistivity, respectively. The current density and fluid vorticity are given by

$$
\mathbf{J}=\boldsymbol{\nabla} \times \mathbf{B}, \quad \boldsymbol{\omega}=\boldsymbol{\nabla} \times \mathbf{v} .
$$

We adopt a planar geometry in which $z$ is the ignorable coordinate. The conservation equations $\boldsymbol{\nabla} \cdot \mathbf{B}=0, \boldsymbol{\nabla} \cdot \mathbf{v}=0$ are satisfied by taking the flux and streamfunction representations,

$$
\mathbf{B}(x, y)=\boldsymbol{\nabla} \psi \times \hat{\mathbf{z}}+b \hat{\mathbf{z}}, \quad \mathbf{v}(x, y)=\boldsymbol{\nabla} \phi \times \hat{\mathbf{z}}+w \hat{\mathbf{z}} .
$$

The planar field components can be isolated by taking the curl of the momentum equation. Explicitly

$$
\begin{aligned}
& {\left[\nabla^{2} \phi, \phi\right]=\left[\nabla^{2} \psi, \psi\right]+\nu \nabla^{4} \phi,} \\
& E+[\psi, \phi]=\eta \nabla^{2} \psi,
\end{aligned}
$$

while the $z$ components are given by

$$
\begin{aligned}
& {[w, \phi]=[b, \psi]+\nu \nabla^{2} w,} \\
& {[b, \phi]+[\psi, w]=\eta \nabla^{2} b,}
\end{aligned}
$$


where $[\psi, \phi]$ is the Poisson bracket,

$$
[\psi, \phi]=\psi_{x} \phi_{y}-\psi_{y} \phi_{x} .
$$

The planar reconnection problem is now specified by three parameters: $\eta, \nu$, and $E$. On the Sun's surface the dimensionless plasma resistivity $\eta$ is very small—of $O\left(10^{-10}\right)$ in the present units-implying that the resistive effect will only be significant in areas of high current density. Since the viscous coefficient $\nu$ is $O(\eta)$ for a collisional plasma, we expect the inclusion of plasma viscosity to have little effect on the results of $\mathrm{CH}$, except in regions where the velocity is highly sheared. The parameter $E$ defines the magnitude of the uniform electric field that drives the external plasma flow. Fast reconnection requires that, for well-defined conditions on the inflow boundary $x=1$ (say), $E$ can be chosen independently of $\eta$ and $\nu$. For example, in classical SweetParker merging, ${ }^{1} \nu=0$ and $E$ scales as $\eta^{1 / 2}$. This dependence qualifies the reconnection rate as slow.

\section{B. Analytic reconnection solutions}

The planar analysis of $\mathrm{CH}$ can be applied in the case of inviscid planar reconnection $\nu=0$. Following $\mathrm{CH}$ we note that the Poisson brackets in Eq. (5) vanish in the case of one-dimensional or harmonic functions. However, if we consider superpositions of the form

$$
\psi=\beta H(x, y)+g(x), \quad \phi=\alpha H(x, y)+f(x),
$$

where $H$ is harmonic, there are nonlinear contributions that prevent the Poisson brackets from vanishing. $\mathrm{CH}$ show that, of all the planar harmonic functions, only $H=x y$ provides an admissible solution.

Motivated by these considerations, we assume reconnection solutions of the form

$$
\begin{aligned}
& \psi=\beta x y+g(x), \quad \phi=\alpha x y+f(x), \\
& b=b(x), \quad w=w(x) .
\end{aligned}
$$

Substituting these expressions into Eqs. (5)-(8) gives

$$
\begin{aligned}
& \left(\alpha f^{\prime \prime \prime}-\beta g^{\prime \prime \prime}\right) x=\nu f^{\prime \prime \prime}, \\
& E+\left(\alpha g^{\prime}-\beta f^{\prime}\right) x=\eta g^{\prime \prime}, \\
& \left(\alpha w^{\prime}-\beta b^{\prime}\right) x=\nu w^{\prime \prime}, \\
& \left(\alpha b^{\prime}-\beta w^{\prime}\right) x=\eta b^{\prime \prime} .
\end{aligned}
$$

In the case of an inviscid plasma, solutions can be expressed in terms of named functions, as discussed below.

\section{INVISCID RECONNECTION}

\section{A. Analytic solution}

For $\nu=0$, Eqs. (12) and (14) imply

$$
f(x)=\frac{\beta}{\alpha} g(x)+q(x), \quad w(x)=\frac{\beta}{\alpha} b(x)+w_{0},
$$

where $q(x)$ is an arbitrary quadratic function and $w_{0}$ is a constant. The effect of $q(x)$ is to add a global external shear to the velocity profile. The influence of global shear flows have been discussed by Besser et al. ${ }^{10}$ within the context of magnetic annihilation models. Since such flows are the least significant feature of the present reconnection solutions, we elect to simplify the discussion by taking $q(x)=0$. By the same token we dismiss as physically uninteresting, the possibility of superposing a uniform component on $w(x)$.

Under these assumptions Eqs. (13) and (15) reduce to

$$
\begin{aligned}
& E+\left(\frac{\alpha^{2}-\beta^{2}}{\alpha}\right) x g^{\prime}=\eta g^{\prime \prime}, \\
& \left(\frac{\alpha^{2}-\beta^{2}}{\alpha}\right) x b^{\prime}=\eta b^{\prime \prime} .
\end{aligned}
$$

Introducing

$$
\begin{aligned}
& \operatorname{daw}(x)=\int_{0}^{x} \exp \left(t^{2}-x^{2}\right) d t, \\
& \operatorname{erf}(x)=\frac{2}{\sqrt{\pi}} \int_{0}^{x} \exp \left(t^{2}\right) d t, \quad \mu^{2}=\frac{\left(\beta^{2}-\alpha^{2}\right)}{2 \alpha \eta},
\end{aligned}
$$

the solution can be written as

$$
\begin{aligned}
& g(x)=\frac{E}{\eta \mu} \int_{0}^{x} \operatorname{daw}(\mu s) d s, \\
& b(x)=\frac{\sqrt{\pi}}{2 \mu} b^{\prime}(0) \operatorname{erf}(\mu x)+b(0) .
\end{aligned}
$$

The magnetic field and the velocity profile are thus given by

$$
\mathbf{B}=\beta(x \hat{\mathbf{x}}-y \hat{\mathbf{y}})-\mathbf{Q}(x), \quad \mathbf{v}=\alpha(x \hat{\mathbf{x}}-y \hat{\mathbf{y}})-\frac{\beta}{\alpha} \mathbf{Q}(x),
$$

where

$$
\mathbf{Q}(x)=\frac{E}{\eta \mu} \operatorname{daw}(\mu x) \hat{\mathbf{y}}-\left(\frac{\sqrt{\pi}}{2 \mu} b^{\prime}(0) \operatorname{erf}(\mu x)+b(0)\right) \hat{\mathbf{z}} .
$$

The key feature of the solution is the presence of a global current sheet in the plane $x=0$. In the limit $\beta=b(x)=0$, the solution reduces to the simple merging of antiparallel field lines, as proposed by Parker ${ }^{15}$ and Sonnerup and Priest. ${ }^{8}$ The fact that reconnection only occurs for nonvanishing $\beta$ shows that the merging of curved field lines is associated with strong shearing motions aligned to the current sheetthat is, a breakdown of the traditional stagnation point flow symmetry in which there is no flow across any coordinate axis.

A typical solution is shown in Fig. 1. Fluid motion advects curved field lines into the neutral point, where they are cut and reconnected. Plasma swept into the dissipation region is ejected along the sheet, in tandem with the reconnected field lines. The effect of finite $z$ components in the solution is to tilt the field out of the original reconnective plane. This tilt is near constant in the outer region and rapidly diminishes as $x \rightarrow 0$.

\section{B. Dissipation of the normal field component}

We first note that the form of solution (21) only makes sense if $\mu^{2}>0$. Thus, if $|\alpha|>|\beta|$ we must take $\alpha<0$, corre- 


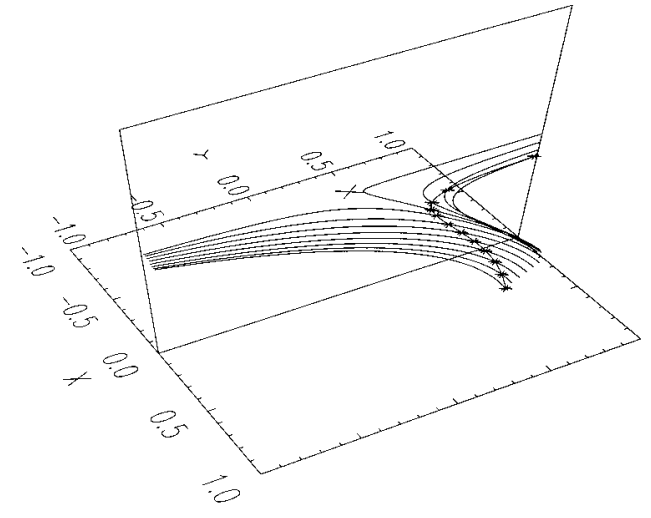

FIG. 1. Two-and-a-half-dimensional solution showing the advection of curved field lines into the neutral point (marked with a cross). The stars represent the inward motion of a typical fluid element prior to expulsion along the sheet. Solution parameters are $\eta=0.01, E=0.1, b^{\prime}(0)=4, b(0)$ $=0, \alpha=-1, \beta=-0.75$.

sponding to inflow along the $x$ axis. Although the flow field washes in both planar and nonplanar components of the magnetic field, only the planar component of the field is amplified as it advects toward the reconnection region. In particular, since $\mu^{-1} \sim \eta^{1 / 2}$ provides the length scale for nonuniformities in the field, $b(x)$ varies rapidly only in a narrow layer overlying the neutral line $x=0$. The fact that $\operatorname{erf}(\mu x)$ is $O(1)$ for large arguments immediately implies that $b(x)$ is constant everywhere except over the current layer.

Let us consider the case in which all planar field components vanish [formally, $E=g(x)=0$ ]. Then the solution reduces to a magnetic annihilation model for the $z$ component of the field, as illustrated in Fig. 2. Despite the presence of nonplanar flows, it is the underlying stagnation flow field $\phi=\alpha x y$ that advects the disturbance field into the current sheet.

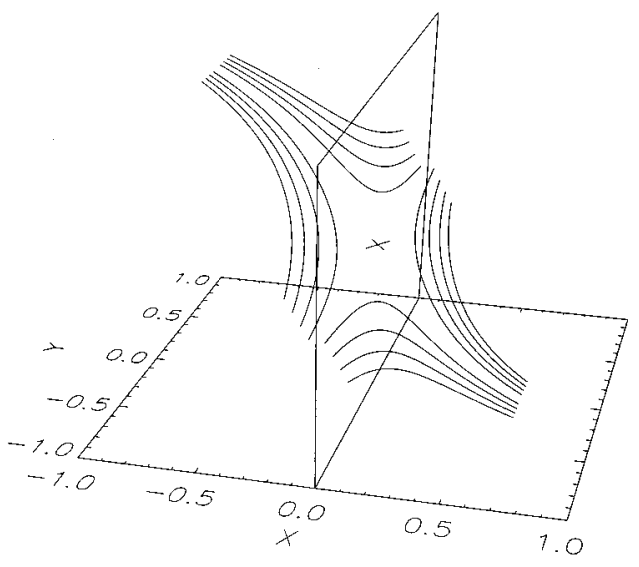

FIG. 2. Field lines for the normal field annihilation solution. The field lines are obtained by superposing the harmonic background field with $b(x) \hat{\mathbf{z}}$. There is no flow across either of the separatrix planes when the planar field component is turned off $[E=g(x)=0]$. Solution parameters are $\eta=0.01$, $E=b(0)=0, b^{\prime}(0)=1, \alpha=-1, \beta=-0.75$.
We now ask what is the dissipation rate of the normal field component? Reconnection scalings are derived by assuming inflow conditions that are independent of $\eta$. Therefore we consider the effect of reducing the plasma resistivity under the assumption that a disturbance field of fixed amplitude, $O(1)$, is washed through the inflow boundary $x=1$ (say). Since $b(x)$ remains of order unity until the onset of the diffusion layer, it follows that $b$ must decrease rapidly toward the neutral line $x=0$-in fact, the current must scale as $\eta^{-1 / 2}$ if a field of unit strength is to be achieved at $x \sim \eta^{1 / 2}$.

Finally, we note that the volume element $d V$ of the current sheet scales as $\eta^{1 / 2}$. Thus, the Ohmic dissipation rate and the flux annihilation rate of the normal field component have the scalings

$$
W_{\eta}=\eta \int J^{2} d V \sim \eta^{1 / 2}, \quad \eta J \sim \eta^{1 / 2}, \quad E=0 .
$$

The field dissipation is nonreconnective, however, in the sense that field lines are nowhere advected across the separatrices of the field. These properties are essentially unchanged by the superposition of the uniform nonplanar component $b(0)$.

\section{Fast dissipation of the planar field}

Consider now the dissipation of the planar component of the field in the case $b(0)=b^{\prime}(0)=0$. The presence of the Dawson function adds a localized shear flow to the background stagnation flow field, which allows curved field lines to be reconnected across the neutral point.

To determine reconnection scalings, we note that the Dawson function, unlike the error function, decreases rapidly in the outer field: $\operatorname{daw}(x)$ declines as $x^{-1}$ for $x \gg 1$. At $x=1$, $|Q| \simeq E / 2 \eta \mu^{2}=\alpha E /\left(\beta^{2}-\alpha^{2}\right)$, and therefore $E$ must scale independently of $\eta$ if the amplitude of the planar field is to be fixed on the outer boundary. Hence, the field achieves a maximum of order $\eta^{-1 / 2}$ at the onset of the current sheet. The flux pile-up of the planar field implies the fast reconnective scalings,

$$
\begin{aligned}
& W_{\eta}=\eta \int J^{2} d V \sim \eta^{-1 / 2}, \quad \eta J \sim \eta^{0}, \\
& b(0)=b^{\prime}(0)=0,
\end{aligned}
$$

Figure 3 shows the effect of equalizing the $y$ and $z$ components of $\mathbf{Q}$ on the inflow boundary $x=1$. The current,

$$
\mathbf{J}=-\left[b^{\prime}(x) \hat{\mathbf{y}}+g^{\prime \prime}(x) \hat{\mathbf{z}}\right],
$$

and hence the vorticity (since $\alpha \boldsymbol{\omega}=\beta \mathbf{J}$ ) are both dominated by the buildup in the planar component of the field. Figure 3 confirms that, at the onset of the diffusion layer, the current is aligned predominantly in the $z$ direction.

We conclude that fast reconnection requires finite planar field components to be washed through the inflow boundary $x=1$. The strong amplification of these components ensures that, even if nonplanar components dominate in the outer field, the merging field lines become increasingly planar as the current layer is approached. The price of driving fast reconnection, however, is the buildup of strong plasma pressures in the outer field. In general, the pressure is given by 


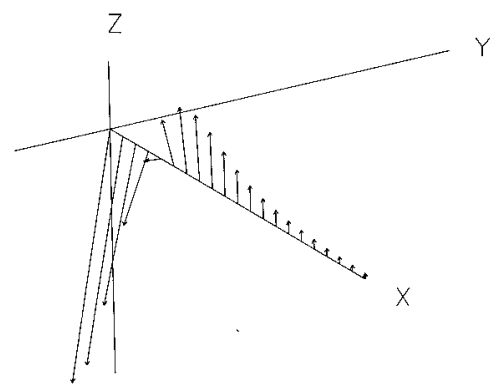

FIG. 3. Current buildup along the $x$ axis with the "perturbation" field $O(1)$. Although both planar and normal field components are comparable on the inflow boundary, the planar field is preferentially magnified as it is advected into the current sheet. Accordingly, the current at $x=0$ is primarily in the $z$ direction. Solution parameters are $\eta=0.01, \alpha=-1, \beta=-0.75$.

$$
p=p_{0}-\frac{1}{2}\left(u_{H}^{2}+Q^{2}\right)-\beta y g^{\prime}(x),
$$

where $\mathbf{u}_{H}=(\alpha x,-\alpha y)$. But we have just seen that the magnitude of $\mathbf{Q}$ is dominated by the $y$ component of the field (which scales as $\eta^{-1 / 2}$ ). It follows that $p_{0}$ must scale as $\eta^{-1}$ to maintain $p>0$. This restrictive behavior can be avoided only by setting the planar field components to zero. In this case, however, there is no reconnection, only field annihilation of the $z$ component at the slow rate $\eta^{1 / 2}$.

Finally, we mention that the extreme scaling on the plasma pressure can be eased slightly by generalizing the solution to 3-D. ${ }^{13}$ Fast dissipation of reconnecting fields can then occur at the rate $W_{\eta} \sim \eta^{0}$ and the scaling of the pressure is reduced to $\eta^{-1 / 2}$. More generally, it appears that strong pressure inhomogeneities within the reconnection region are common to all flux pile-up solutions (e.g., Forbes and Priest $\left.{ }^{1}\right)$.

\section{SOLUTION FOR NONVANISHING VISCOSITY}

\section{A. Introduction}

The present solution makes clear that reconnection is associated mainly with the magnification of the planar field as it is swept toward the neutral point. Although the vorticity is negligible outside the current sheet, the strong shearing motions across the current layer suggest that viscous effects could be significant in dissipating the energy of the fluid. Since the inner solution is dominated by the flux pile-up of the planar field, and the shearing motions are most severe in planar reconnection, we choose to simplify the calculation by taking $w=b=0$.

A detailed discussion of viscous effects within the context of stagnation flow magnetic annihilation models has been given by Jardine et al. ${ }^{11}$ Clearly, the inclusion of viscosity adds significantly to the mathematical complexity of the problem. This is manifested, as we shall see, by the need to specify more boundary conditions on the dependent variables for $\nu>0$.

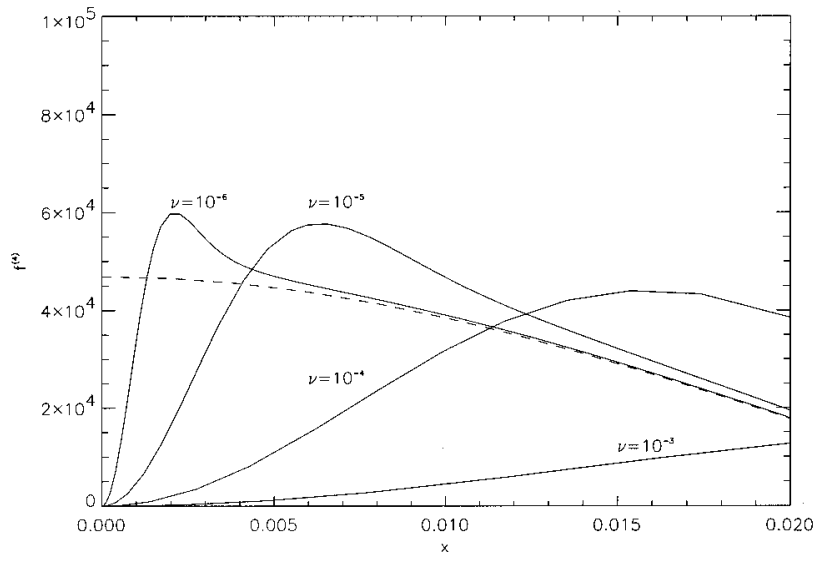

FIG. 4. $f^{\prime \prime \prime \prime}$ for various values of the viscous coefficient $\nu$. Although the graphs merge in the outer field, a significant difference is always maintained within the current layer. In particular, an increasingly sharp transition layer emerges as $\nu \rightarrow 0$. The dashed line represents the unattainable $\nu=0$ limit.

\section{B. Inner series expansion}

Obviously, the inclusion of viscosity cannot undo the inherent symmetry of the inviscid solution. Assuming an inner series expansion of the form

$$
f(x)=\sum_{n}^{\infty} \frac{f_{n}}{n !} x^{n}, \quad g(x)=\sum_{n}^{\infty} \frac{g_{n}}{n !} x^{n},
$$

where $n$ is even, we find that Eqs. (12) and (13) yield the following inner solution:

$$
\begin{aligned}
& f(x)=\frac{\alpha}{\beta} \frac{E}{\eta} \frac{x^{2}}{2}-\frac{g_{4}}{4 !} x^{2}\left(\frac{6 \eta}{\beta}+\frac{\beta}{15 \nu} x^{4}+\cdots\right), \\
& g(x)=\frac{E}{\eta} \frac{x^{2}}{2}+\frac{g_{4}}{4 !} x^{4}\left(1+\frac{2 \alpha}{15 \eta} x^{2}+\cdots\right) .
\end{aligned}
$$

It is clear that, while $g^{\prime \prime}(0)=E / \eta$ is determined by the external parameters of the problem, $g_{4}$ can be chosen arbitrarily, provided that $\nu>0$.

There are fundamental differences between the viscous and inviscid solutions. In the case $\nu=0$ we can express the solution of Sec. III in terms of a hypergeometric series, ${ }^{12}$ viz.,

$g(x)=\frac{E}{\eta} \frac{x_{1}^{2}}{2} F_{2}\left(1, \frac{3}{2}, 2,-\mu^{2} x^{2}\right)=\frac{E}{\eta} \frac{x^{2}}{2}\left(1-\frac{\mu^{2} x^{2}}{3}+\cdots\right)$.

Since $f$ is linearly related to $g, f^{\prime \prime \prime \prime}(0)$ is nonvanishing for the inviscid solution. Equation (28) shows, however, that $f_{4}=0$ and so the vorticity dissipation rate $\left(\nabla^{2} \boldsymbol{\omega}\right)$ always vanishes at the origin for finite $\nu$. To obtain localized current sheets we must take $g_{4}$ finite, and therefore the inviscid relationship $f=(\beta / \alpha) g$ no longer holds. The effect of finite viscosity on $f^{\prime \prime \prime \prime}(x)$ is demonstrated in Fig. 4, where we see that departures from the $\nu=0$ limit are always present close to the origin. 


\section{Numerical results}

Finally, it remains to investigate whether the changes incurred by the inclusion of viscosity compromise the fast reconnection rates of $\mathrm{CH}$. In the absence of an analytic viscous solution we must rely upon numerical simulations to determine reconnection scalings.

We assume that the reconnection region is defined over the unit square $|x|,|y| \leqslant 1$. To determine reconnection rates we fix field amplitudes on the inflow boundaries and systematically vary $\eta$ (keeping $\alpha, \beta$, and $\nu$ constant). At this stage, the system parameter $E$ should be regarded as a function of $\eta$.

The inflow of magnetized plasma is determined by evaluating $[\psi, \phi]$ over the boundary. Since the advection bracket is independent of $y$, and even in $x$, it is sufficient to specify inflow conditions on $x=1$. We have that

$$
[\psi, \phi]_{x=1}=\eta g^{\prime \prime}(1)-E,
$$

which reduces to

$$
\alpha g^{\prime}(1)-\beta f^{\prime}(1) \simeq-E,
$$

if the current is localized at $x=0$. Under these conditions, $E$ must be taken independent of $\eta$ to maintain the rate of material inflow.

For numerical purposes, however, it is easiest to assume inner conditions on $x=0$ and to "shoot" to satisfy the inflow conditions at $x=1$. For nonvanishing viscosity, Eqs. (12) and (13) can be combined to yield a fourth-order equation for the field potential $g(x)$. Appropriate inner conditions are therefore

$$
g=g^{\prime}=g^{\prime \prime \prime}=0, \quad g^{\prime \prime}=\frac{E}{\eta} .
$$

But Eq. (32) makes clear that the amplitude of the disturbance field $g^{\prime}(1)$ is not uniquely determined for $\beta>0$. This ambiguity can be eliminated by normalizing $g^{\prime}(1)$ according to the prescription

$$
g^{\prime}(1)=g_{0}^{\prime}(1),
$$

where $g_{0}^{\prime}(1)$ is the outer boundary value for the $\nu=0$ solution. To achieve this normalization we exploit the fact-as expression (29) confirms-that the coefficient $g_{4}$ can be chosen arbitrarily. Therefore, we systematically shoot using $g_{4}$ until Eq. (34) is satisfied. In this way the inflow conditions of the inviscid calculation are reproduced.

The results of the numerical experiments, subject to Eqs. (33) and (34), are evident in Fig. 5. The Ohmic dissipation rate scales marginally slower than the inviscid model [see Eq. (24)], but the solution is still fast, $W_{\eta} \sim \eta^{-0.48}$. It follows that viscous damping of the flow's inner "shearing layer" does not compromise the global reconnection scalings of the solution.

\section{CONCLUSIONS}

We have considered exact solutions for 2-D planar reconnection. Our analysis shows that fast reconnection is

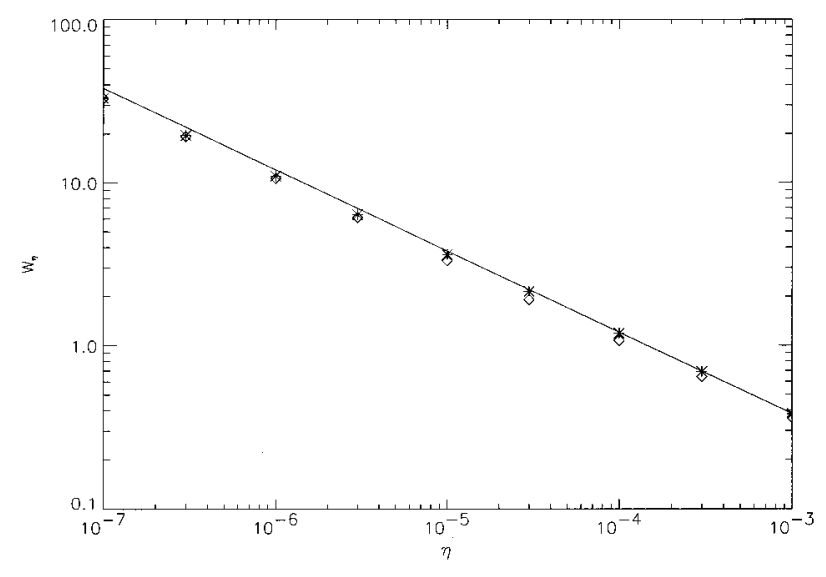

FIG. 5. Scaling of the Ohmic dissipation rate $W_{\eta}$ for various values of the viscous coefficient $\nu$. The filled line shows $\nu=0$, diamonds show $\nu=10^{-2}$, and stars show $\nu=10^{-6}$. Solution parameters are $\alpha=-1, \beta=0.25, E=0.1$.

maintained by the strong magnification of the planar field as it advects toward the current layer. This allows flux annihilation to proceed independently of the plasma resistivity, and the Ohmic dissipation rate to achieve the superfast scaling $\eta^{-1 / 2}$. Nonplanar components of the field, however, are not amplified as they are carried toward the current layer. Accordingly, the dissipation of the perpendicular field occurs only at the slow Sweet-Parker rate $\eta^{1 / 2}$.

Since magnetic reconnection requires the presence of strong shearing motions across the current layer, it is natural to expect that viscous effects will significantly modify the inviscid solutions. Specifically, we find that the Fourier development of the solution across the inner diffusive layer is fundamentally changed for any finite $\nu$-the dissipation rate of the vorticity has to vanish. For fixed inflow conditions, however, the global energy dissipation rates remain essentially unaffected for all physically significant levels of plasma viscosity.

${ }^{1}$ T. G. Forbes and E. R. Priest, Rev. Geophys. 25, 1587 (1987).

${ }^{2}$ D. Biskamp, Phys. Rev. Lett. 237, 181 (1994).

${ }^{3}$ Solar Flare Magnetohydrodynamics, edited by E. R. Priest (Gordon and Breach, London, 1981).

${ }^{4}$ I. J. D. Craig and A. N. McClymont, Astrophys. J. Lett. 371, L41 (1991).

${ }^{5}$ I. J. D. Craig and A. N. McClymont, Astrophys. J. 405, 207 (1993).

${ }^{6}$ I. J. D. Craig and P. G. Watson, Astrophys. J. 393, 385 (1992).

${ }^{7}$ A. B. Hassam, Astrophys. J. 399, 159 (1992).

${ }^{8}$ B. U. Ö. Sonnerup and E. R. Priest, J. Plasma Phys. 14, 283 (1975).

${ }^{9}$ F. T. Gratton, M. F. Heyn, H. K. Biernat, R. P. Rijnbeek, and G. Gnavi, J. Geophys. Res. 93, 7318 (1988).

${ }^{10}$ B. P. Besser, H. K. Biernat, and R. P. Rijnbeek, Planet. Space Sci. 38, 411 (1990).

${ }^{11}$ M. Jardine, H. R. Allen, R. E. Grundy, and E. R. Priest, J. Geophys. Res. 97, 4199 (1992).

${ }^{12}$ I. J. D. Craig and S. M. Henton, Astrophys. J. 450, 280 (1995).

${ }^{13}$ I. J. D. Craig, R. B. Fabling, S. M. Henton, and G. J. Rickard, Astrophys. J. Lett. 455, L197 (1995).

${ }^{14}$ I. J. D. Craig and R. B. Fabling, Astrophys. J. 462 (1996).

${ }^{15}$ E. N. Parker, Astrophys. J. Suppl. Ser. 8, 177 (1963). 